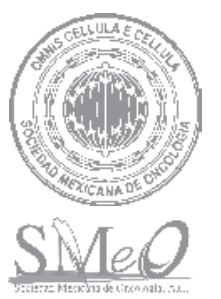

SOCIEDAD MEXICANA DE ONCOLOGÍA, A.C.

\title{
La medicina nuclear y el cáncer diferenciado de tiroides
}

\author{
Sevastián S. Medina-Ornelas ${ }^{1, *}$ Francisco O. García-Pérez ${ }^{1}$ y Martín Granados-García ${ }^{2}$
}

\author{
${ }^{1}$ Departamento de Medicina Nuclear e Imagen; ${ }^{2}$ Departamento de Cirugía de Cabeza y Cuello. Instituto Nacional de \\ Cancerología, Ciudad de México, México
}

Recibido el 5 de junio de 2017; aceptado el 8 de agosto de 2017

Disponible en Internet el 4 de diciembre de 2017

\section{PALABRAS CLAVE \\ Cáncer de tiroides; \\ Radioyodo; \\ Medicina nuclear; \\ Tratamiento}

\section{KEY WORDS}

Thyroid cancer;

Radioiodine;

Nuclear medicine;

Treatment

\begin{abstract}
Resumen: Los afectados por cáncer diferenciado de tiroides (CDT) suelen tener un curso clínico favorable, pero algunos pueden mostrar un curso agresivo con recaídas. El tratamiento óptimo aún es controvertido, en especial respecto a la extensión de la cirugía, las indicaciones de radioyodo y la supresión de hormona estimuladora del tiroides (TSH). La evaluación correc ta de los riesgos facilita un enfoque selectivo de la terapia. Presentamos una revisión del papel de la medicina nuclear en la evaluación, tratamiento y seguimiento de pacientes afectados por esta interesante enfermedad. (creativecommons.org/licenses/by-nc-nd/4.0/).
\end{abstract}

\begin{abstract}
Those affected by differentiated thyroid cancer tend to have a favorable clinical course, but some may show an aggressive course with relapses. The optimal treatment is still controversial, especially regarding the extent of surgery, indications for radioiodine and suppression of thyroid-stimulating hormone. Correct risk assessment facilitates a selective approach to therapy. We present a review of the role of nuclear medicine in the evaluation; treatment and follow-up of patients affected by this interesting disease.
\end{abstract}

\section{INTRODUCCIÓN}

Según Globocan, en 2012 en México ocurrieron 3,036 casos de cáncer de tiroides (725 en hombres y 2,311 en mujeres), que constituyeron el $2.1 \%$ del total de neoplasias malignas ${ }^{1}$. El CDT representa al menos el $85 \%$ de los casos e incluye los tipos papilar, folicular y de células de Hürtle. El cáncer papilar, el tipo más frecuente, se asocia a una excelente supervivencia, pero algunos pacientes tienen un curso clínico menos favorable, con metástasis o recaídas que afectan a los ganglios linfáticos cervicales y, con menor frecuencia, a los pulmones y el hueso ${ }^{2,3}$.

La medicina nuclear se sirve de las peculiaridades de la fisiopatología del CDT y juega un papel muy importante tanto en el tratamiento adyuvante como en el seguimiento y manejo de las recaídas. El tratamiento debe ser el más eficaz posible, con las menores secuelas y el menor costo por ello se enfatiza el tratamiento individualizado, acorde al tipo histológico, la extensión de la enfermedad y el riesgo de recaída.

\section{YODO 131}

El yodo 131 ([131]l o radioyodo) es producido en un reactor nuclear mediante la irradiación neutrónica de dióxido de te lurio durante la fisión del uranio. Su vida media física es de 8.02 días. Los átomos del yodo 131 emiten partículas $B$ con

*E-mail para correspondencia: dr.sevastian@outlook.com (S.S. Medina-Ornelas) 
varias energías, la máxima de $606 \mathrm{keV}$, fundamental para el efecto terapéutico del yodo 131. Después de la emisión de la partícula $B$, emite rayos $\gamma$, con energía de 364 a 637 keV, útil para obtener imágenes gammagráficas o mediante tomografía computarizada de emisión monofónica (SPECT). El yodo 131 se dispensa en forma de yoduro líquido o en cápsulas para su ingesta oral ${ }^{4}$.

\section{RADIOBIOLOGÍA}

La mayor radiación emitida por el [131] l ocurre en la forma de partículas $B$ que interrumpe los enlaces químicos e inflige un daño devastador al ADN, produciendo una disfunción celular y finalmente la activación de la muerte celular programada. Esto sucede mediante dos vías: la primera, o directa, resulta de la irradiación de las partículas $B$ sobre la cadena simple del ADN, mientras que la segunda, o indirecta, ocurre mediante la interacción con moléculas de agua formando radicales libres, que dañan al $A D N$; esta última vía es la más importante debido al mayor daño infringido ${ }^{5}$.

Las partículas $B$ penetran en el tejido un par de milímetros. La dosis absorbida y entregada por las partículas $B$ para una determinada dosis radioactiva aumenta con el radio de tejido hasta los $10 \mathrm{~mm}$ y luego permanece constante. Debido a que las partículas $B$ virtualmente no escapan de depósitos tumorales grandes, pueden suministrarse grandes dosis de yodo 131 sin dañar los tejidos circundantes. La falta de homogeneidad en la dosis de radiación se debe a la distribución irregular del yodo 131 en los focos neoplásicos y al pequeño radio de acción de las partículas $B$.

La radiación y aporta solamente el $10 \%$ de radiación total, y sólo una pequeña fracción de rayos $\gamma$ son absorbidos por el tejido funcional, mientras que la mayor parte abandona al paciente a través de su superficie; esta radiación puede detectarse para convertirse en imágenes y es responsable de que el paciente deba permanecer aislado cuando se suministran dosis mayores a $30 \mathrm{mCi}$, conforme la legislación internacional vigente ${ }^{6}$.

La eficacia del yodo 131 es directamente proporcional a la captación y retención por el tejido tumoral. La captación tumoral efectiva ocurre si alcanza el $0.5 \%$ de la dosis de yodo 131 por gramo de tejido y tiene una vida media biológica de cuatro días. Con una dosis de $150 \mathrm{mCi}$ (equivalente a $5.6 \mathrm{GBq}$ ) de yodo 131 el tumor puede recibir 25,000 cGy, que equivale a cuatro veces la dosis liberada por una terapia de radiación de haz externo ${ }^{7}$.

\section{BIOLOGÍA}

El yodo es esencial para la síntesis de hormonas tiroideas. Después de su ingesta es reducido a yoduro en el intestino delgado proximal. Más del $90 \%$ es absorbido durante los primeros $60 \mathrm{~min}$. Se distribuye en la sangre como un ion extracelular, similar al cloruro, para incorporarse a las células gracias al cotransportador $\mathrm{Na}^{+} / \mathrm{I}^{-}(\mathrm{NIS})^{8}$.

El NIS es una proteína de la membrana basolateral de la célula folicular que cotransporta dos iones de sodio y un ion yoduro. El gradiente de sodio provee la energía para esta transferencia, y es mantenido por la bomba $\mathrm{Na}^{+} / \mathrm{K}^{+}$-ATPasa. En el interior de la célula, el yodo penetra la membrana api- cal hasta el coloide, gracias a transportadores como la pendrina. La tiroperoxidasa (TPO) de la membrana apical actúa sobre el yodo mediante tres pasos secuenciales: primero lo oxida, luego lo incorpora a los residuos de tirosina de la tiroglobulina ( $\mathrm{Tg}$ ) y finalmente cataliza el acoplamiento de dos residuos de tirosina yodados para formar las futuras hormonas tiroideas. Este proceso se denomina organificación del yodo?.

Luego, dependiendo de la necesidad de hormonas tiroideas del organismo, la Tg es endocitada por la membrana apical en forma de gotas de coloide que son degradadas por enzimas lisosomales, liberando triyodotironina y tiroxina (T3 y T4, respectivamente) al torrente sanguíneo. Este pro ceso es regulado por la TSH, que, tras unirse a su receptor (TSH-r) en la membrana basolateral, activa las vías depen dientes del AMPC e induce el transporte de yodo por regulación de la expresión de NIS, tanto transcripcional como postranscripcional (Fig. 1).

\section{Distribución normal del cotransportador $\mathrm{Na}^{+} / \mathrm{I}$}

Además de en el tejido tiroideo, se ha detectado mRNA-NIS en las glándulas salivales, el estómago, el timo, las mamas $y$, en bajos niveles, en la próstata, el ovario, las glándulas adrenales, el pulmón y el corazón ${ }^{10}$. Esto es consistente con la captación normal de yodo 131 en las glándulas salivales, el estómago, la mama lactante y la placenta. Ocasionalmente la absorción de yodo 131 también se observa en mamas no lactantes ${ }^{11}$.

\section{Expresión del cotransportador $\mathrm{Na}^{+} / l^{-}$en el carcinoma tiroideo}

Usando inmunohistoquímica con anticuerpos anti-NIS se detecta NIS en menor proporción en los tejidos tiroideos neoplásicos que en los normales, y se demuestra una expresión heterogénea en las células malignas del carcinoma papilar o folicular. En los tejidos tiroideos neoplásicos la expresión de mRNA-NIS es de 10 a 1,200 veces menor que en el tejido tiroideo normal; la expresión de mRNA-TPO está reducida en 5-500 veces, mientras que la expresión de mRNA-Tg es 300 veces menor ${ }^{12}$. Dohan, et al. ${ }^{13}$ reportaron que el $70 \%$ de los

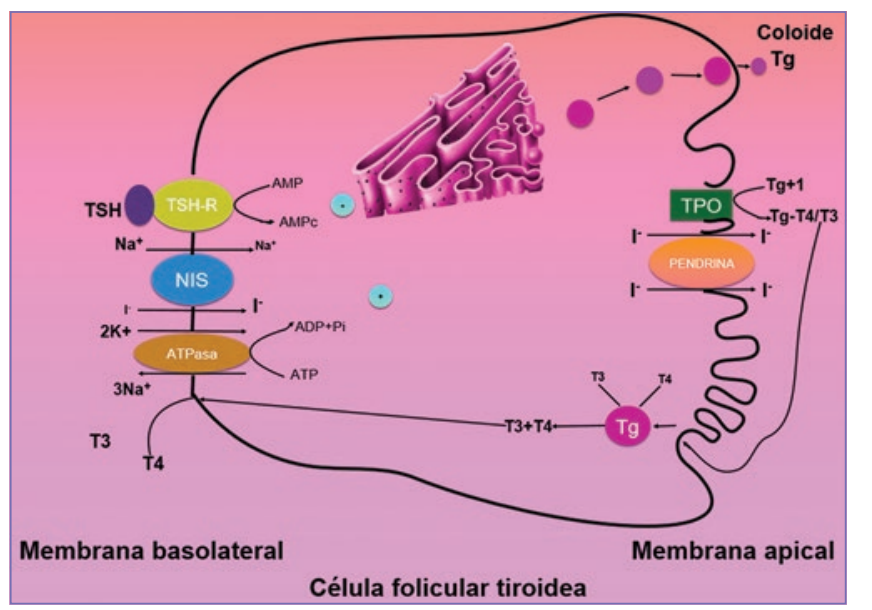

Figura 1. El NIS transporta yodo junto con dos átomos de sodio de forma activa al interior de la célula por la membrana basolateral; una vez en su interior, el yodo pasa por la membrana apical al coloide, gracias a la pendrina. Todo este proceso es regulado por la TSH. 
tumores tiroideos muestran un incremento en la expresión intracelular del NIS respecto al tejido tiroideo sano. Estos resultados discordantes se explican por diferencias metodológicas, pero también sugieren que el NIS se sobreexpresa en las células neoplásicas, aunque la transformación maligna interfiere con la correcta localización del NIS en la célula, lo que deteriora su función ${ }^{14}$.

Además, la mayoría de los investigadores documenta que la expresión del NIS guarda una relación inversa con la diferenciación de las células neoplásicas. El NIS es expresado siempre en los cánceres más diferenciados, mientras que en la mayoría de los pobremente diferenciados es negativo ${ }^{15}$. La localización del transportador en la membrana es indispensable para que sea funcional y capte el yodo. No basta con que el NIS se exprese, debe ubicarse en la membrana celular para que sea plenamente funcional ${ }^{16}$.

\section{ABLACIÓN Y TRATAMIENTO CON YODO 131}

La captación del yodo 131 ocurre bajo la influencia de la TSH y la activación de AMPc, pero otros factores también influyen: la insulina, el factor de crecimiento insulínico tipo $1 \mathrm{y}$ el factor de crecimiento epidérmico. Sin embargo, la TSH es la principal responsable de dicha captación, mientras que AMPc incrementa la biosíntesis del NIS.

Una medida eficaz consiste en evitar contrastes radiográficos yodados durante tres meses y cualquier medicación con alto contenido en yodo, como amiodarona, multivitamínicos, antibióticos (penicilina), jarabes para la tos (ambroxol/ dextrometorfano) e incluso algunos cosméticos que contienen elevadas cantidades de yodo, ya que pueden interferir en la acción del yodo $131^{17}$.

\section{ESTRATEGIAS PARA ELEVAR LA HORMONA ESTIMULADORA DEL TIROIDES}

Para estimular la captación de yodo 131 por las células tumorales usualmente se elimina la medicación supresora con levotiroxina para elevar la TSH endógena o se administra TSH recombinante humana (rhTSH) (Thyrogen $\left.{ }^{\circledR}\right)$. Esto supone que las células neoplásicas conservan cierta diferenciación y respuesta hormonal. Ocasionalmente el cáncer de tiroides pierde esta capacidad asociándose a un pronóstico considerablemente deteriorado ${ }^{18}$.

Después de la tiroidectomía total los niveles de T3, T4 y TSH son inicialmente normales y 2-3 semanas después los de TSH comienzan a elevarse mientras que los de T3 y T4 disminuyen. Generalmente se evita el tratamiento sustitutivo hormonal las primeras 4-6 semanas desde la tiroidectomía si se ha decidido, con base en la evaluación de riesgos, administrar yodo 131. Sin embargo, otras instituciones, debido a logística, inician de inmediato la terapia sustitutiva hormonal ${ }^{19}$.

La elevación de TSH por encima de $30 \mathrm{mU} / \mathrm{l}$ es necesaria para obtener la suficiente concentración de yodo 131 en las células que expresan NIS. Suspender o evitar la terapia sustitutiva hormonal por cuatro semanas en tiroidectomizados abate los niveles de T3 y T4 y eleva los de $\mathrm{TSH}^{20}$. En consecuencia, los pacientes se exponen a un periodo prolongado de hipotiroidismo y síntomas asociados, con un deterioro en la calidad de vida y probabilidad de crecimiento tumoral ${ }^{21}$.
Una alternativa para evitar el estado hipotiroideo causado por la suspensión de LT4 incluye el uso de rhTSH, que tiene una estructura idéntica a la TSH humana, pero su glucosilación difiere, y tiene un mayor contenido de ácido siálico, lo que se asocia a menor actividad inmunológica, menor afinidad por el TSH-r y menor bioactividad in vitro que la TSH humana.

En pacientes con CDT y función renal conservada que reciben $0.9 \mathrm{mg}$ de rhTSH tiene una vida media de $22 \mathrm{~h}$. Las concentraciones de T3 y T4 se incrementan 54 y $89 \%$, respectivamente, entre las 4 y las $8 \mathrm{~h}$ subsecuentes a la administración de la rhTSH, mientras que el pico de Tg ocurre a las 48 $\mathrm{h}^{22}$. Un estudio comparó la tasa de exposición a la radiación entre individuos sometidos a supresión hormonal versus admi-g nistración de rhTSH y demostró que con el segundo procedimiento la exposición a la radiación es menor y más corta, lo que da como resultado una menor concentración de Tg y una menor captación de yodo 131 por las células tumorales ${ }^{23,24}$.

La rhTSH es muy eficaz, pero relativamente costosa. Una tercera estrategia consiste en sustituir LT4 por LT3, que tiene una vida media mucho menor: 1-3 días (en lugar de los siete días de la LT4), y por ello puede suspenderse por menos tiempo para elevar los niveles de $\mathrm{TSH}^{25}$. Los pacientes que suspenden LT4 y la sustituyen por LT3 se someten sólo a dos semanas de hipotiroidismo, mientras que con LT4 son cuatro semanas.

Se ha señalado la necesidad de practicar una dieta baja en yodo durante las dos semanas previas a la administración de yodo 131, para obtener una yoduría $<50 \mu \mathrm{g} / \mathrm{gCr}^{26}$. Esto se basa en viejos estudios en los que se demostró que luego de una dieta baja en yodo la captación por las células tumorales se incrementaba al doble; sin embargo, no hay estudios que examinen si una dieta baja en yodo impacta en la tasa de recaída o mortalidad ${ }^{27}$.

La rhTSH se reserva para casos especiales donde el estado hipotiroideo puede agravar una condición médica subyacente, como el síndrome metabólico mal controlado, la enfermedad renal crónica (tasa de filtrado glomerular [TFG] $<50 \mathrm{mg} / \mathrm{ml}$ ), la depresión mayor, la arritmia cardíaca (en particular si se está en tratamiento con amiodarona y ésta es insustituible) o en pacientes con tejido residual y/o metastásico voluminoso que impida la elevación de TSH debido a la producción continua de hormonas tiroideas ${ }^{28}$.

La guía de la British Thyroid Association (BTA) sugiere que en pacientes con T1-T3, N0, N1a, M0 y R0 el método de elección es administrar rhTSH, pero en pacientes de alto riesgo (véase más adelante), en recaída, con enfermedad metastásica, el método de elección debe ser suspender LT4; sin embargo, se requieren estudios prospectivos que susten-8 ten dicha recomendación.

Independientemente de la estrategia empleada, se debe corroborar que los niveles de TSH sean óptimos al recibir yodo 131, es decir, que estén por encima de $30 \mathrm{mU} / \mathrm{l}$; suele medirse en conjunto con la $\mathrm{Tg}$ y los anticuerpos antitiroglo bulina (Ac-anti-Tg) ${ }^{29}$ (Fig. 2).

\section{EVALUACIÓN DEL RIESGO}

Aún existen controversias relativas a la ablación con radioyodo, pero se aduce que administrar yodo 131 simplifica el seguimiento al eliminar el tejido tiroideo normal o neoplá- 


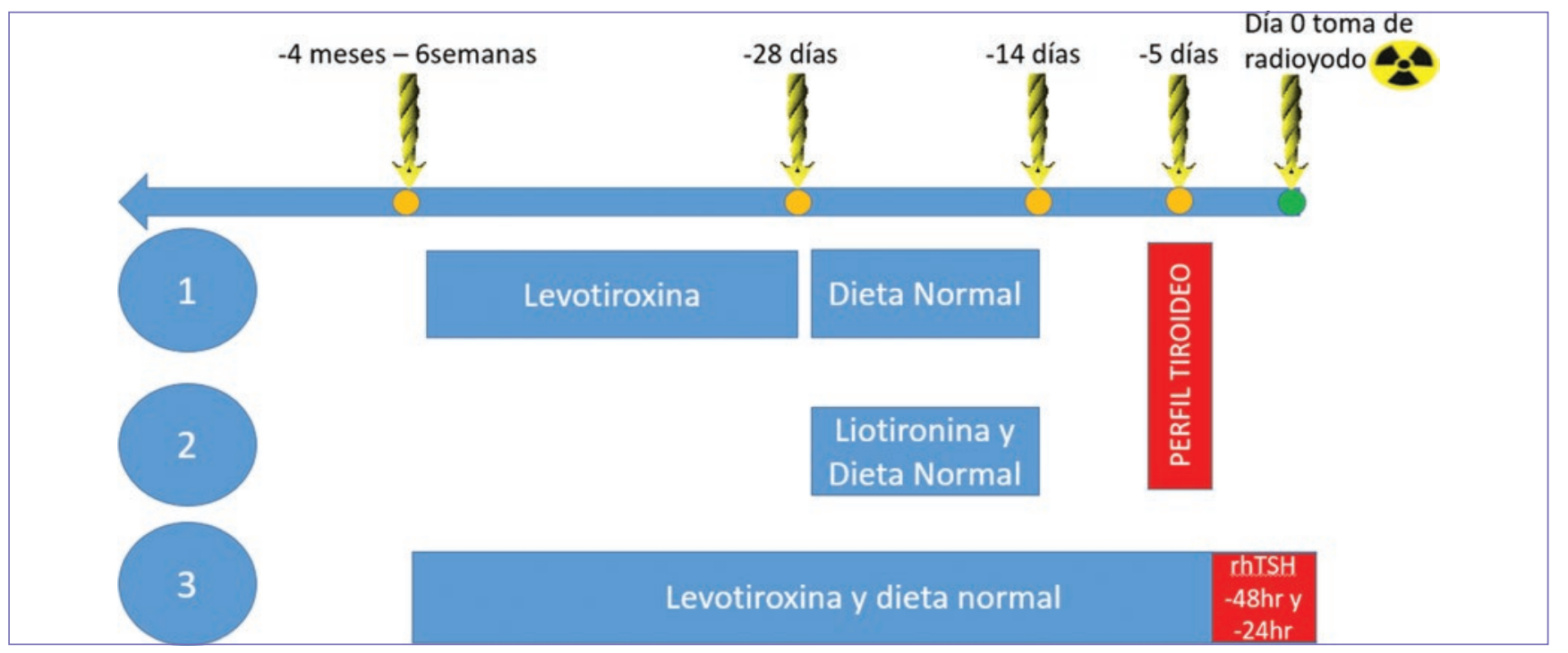

Figura 2. Estrategias para elevar la TSH. Con el fin de lograr niveles óptimos para recibir yodo 131 se pueden seguir tres estrategias: la primera consiste en suspender la levotiroxina cuatro semanas previas a la administración de yodo 131; en la segunda se intercambia levotiroxina por liotironina, la cual se suspende dos semanas antes del yodo 131; por último, se puede optar por administrar rhTSH sin suspender las hormonas tiroideas.

sico productor de Tg, lo que facilita la identificación de recaídas midiendo la $\mathrm{Tg}$ y evitando la producción continua de Ac-anti- $\mathrm{Tg}^{30}$. La decisión de suministrar dosis ablativas debe ser racional, basada en evaluaciones del riesgo. Las guías de la American Thyroid Association (ATA) (2015) y la BTA (2014) proponen sistemas similares, e incluso la National Comprehensive Cancer Network (NCCN) (V1.2016) concuerda en varios puntos ${ }^{31,32}$ (Tabla 1).

La guía ATA surgió de la incapacidad de otros sistemas para establecer el riesgo de recaída; los sistemas previos sólo son útiles para establecer el riesgo de mortalidad. Es un hecho que un paciente de bajo riesgo de mortalidad puede tener alto riesgo de recaída; por tal motivo, la ATA, la BTA y la NCCN recomiendan realizar la evaluación del riesgo de recaída en todos los pacientes.

\section{¿Quiénes deben recibir yodo 131 y cuál es la dosis apropiada?}

El objeto de administrar yodo 131 es eliminar cualquier depósito microscópico de carcinoma tiroideo y tejido tiroideo normal. La NCCN y la ATA proponen tres objetivos para su administración: ablación del tejido tiroideo remanente (el término ablación sólo debe emplearse cuando se administra la primera dosis de yodo 131 para simplificar el seguimiento); terapia adyuvante, para eliminar las micrometástasis subclínicas, y dosis terapéutica, cuyo objetivo es tratar la persistencia local o las metástasis confirmadas. Sin embargo, para fines prácticos, la aplicación de yodo 131 suele clasificarse en ablación y tratamiento. La eficiencia del yodo 131 es inversamente proporcional al volumen de tejido tiroideo residual y directamente proporcional a los niveles de $\mathrm{TSH}$, por lo que debe eliminarse quirúrgicamente toda la enfermedad macroscópica ${ }^{33}$.

A la fecha existen tres enfoques para definir la dosis de yodo 131: dosis fijas, dosis determinada por el límite superior de la sangre y dosimetría tumoral cuantitativa. Estos últimos a menudo se reservan para pacientes con metástasis o situaciones inusuales, como insuficiencia renal, niños, ancianos y pacientes con extensas metástasis pulmonares. Debido a la complejidad de los dos últimos métodos, se favorece la política de dosis fijas ${ }^{34}$.

\section{Pacientes de riesgo bajo}

Estudios prospectivos del Nacional Thyroid Cancer Treatment Cooperative Study Group (NTCTCSG) sugieren que la supervivencia libre de progresión y la supervivencia globat enfermedad específica no mejoran con el tratamiento con yodo 131 en pacientes en estadios I o II. Un estudio retros pectivo multicéntrico, donde se evaluaron 1,298 pacientes de bajo riesgo, con un seguimiento promedio de 10.3 años, no encontró beneficios de la ablación con yodo 131 en relación con la supervivencia global y la supervivencia libre de progresión ${ }^{35}$. En concreto, existe poca evidencia que apoye el uso de yodo 131 en estos pacientes.

Aunque no existen ventajas en términos de supervivencia, ciertos estudios han demostrado la conveniencia de dosis bajas versus altas en pacientes de bajo riesgo, independientemente del método de estimulación ${ }^{36}$. Cheng, et al. ${ }^{37}$ analizaron nueve estudios aleatorizados, que incluyeron 2,569 pacientes de bajo riesgo y diferentes métodos de estimulación de TSH (rhTSH y suspensión hormonal). No encontraron diferencias en la eficacia del yodo 131 entre 30 y $100 \mathrm{mCi}$.

La ablación con $30 \mathrm{mCi}$ o con $100 \mathrm{mCi}$ son equivalentes, independientemente del método de estimulación, con las ventajas de que las dosis no mayores de $30 \mathrm{mCi}$ no requieren hospitalización, la calidad de vida es similar y los efectos secundarios relacionados con el yodo 131, como sialoadenitis, edema, náusea y vómito, son menores con las dosis bajas; además, hay menor tasa de exposición a radiación y menor dosis acumulada. Recientes análisis y revisiones sistemáticas apoyan estas conclusiones ${ }^{38}$.

\section{Pacientes de riesgo intermedio}

Esta categoría causa mayor confusión debido a las discrepancias en la literatura ${ }^{39}$. La administración de yodo 131 
Tabla 1. Grupos de riesgo de acuerdo a la ATA

\begin{tabular}{|c|c|c|}
\hline Riesgo bajo & Riesgo intermedio & Riesgo alto \\
\hline $\begin{array}{l}\text { Todos los siguientes están presentes: } \\
\text { - Sin metástasis } \\
\text { - Resección completa } \\
\text { - Sin extensión extratiroidea } \\
\text { - Sin histología agresiva } \\
\text { - Sin invasión vascular } \\
\text { - Sin captación fuera del lecho } \\
\text { tiroideo postablación con yodo } 131 \\
\text { - N0, < } 5 \text { N1 micrometástasis } \\
\text { - Variante folicular encapsulada, } \\
\text { intratiroidea } \\
\text { - Carcinoma folicular bien } \\
\text { diferenciado, intratiroideo, con } \\
\text { invasión capsular mínima y sin o con } \\
\text { mínima invasión vascular (< } 4 \text { focos) } \\
\text { - Microcarcinoma papilar } \\
\text { intratiroideo, uni o multifocal, sin } \\
\text { mutación de BRAFV600E }\end{array}$ & $\begin{array}{l}\text { Alguno de los siguientes está presente: } \\
\text { - Extensión extratiroidea } \\
\text { microscópica } \\
\text { - Captación de yodo } 131 \text { fuera del } \\
\text { lecho tiroideo luego de ablación de } \\
\text { remanentes } \\
\text { - Histología agresiva (células altas, } \\
\text { insular, columnares, en tachuela, } \\
\text { de Hulte) } \\
\text { - Carcinoma papilar con invasión } \\
\text { vascular } \\
\text { - N1 patológico (> } 5 \text { ganglios } \\
\text { patológicos) con dimensión global } \\
\text { no mayor a } 3 \text { cm } \\
\text { - Carcinoma papilar multifocal con } \\
\text { extensión extratiroidea mínima y } \\
\text { mutación de BRAFV600E }\end{array}$ & $\begin{array}{l}\text { Alguno de los siguientes está } \\
\text { presente: } \\
\text { - Extensión extratiroidea } \\
\text { macroscópica } \\
\text { - Resección incompleta del } \\
\text { tumor (R2) } \\
\text { - Metástasis } \\
\text { - Niveles elevados de Tg pos-Qx } \\
\text { - N1 patológico con cualquier } \\
\text { número de ganglios metastásicos } \\
\text { o dimensión global mayor a } 3 \mathrm{~cm} \\
\text { - Carcinoma folicular con invasión } \\
\text { vascular extensa (> } 4 \text { focos) }\end{array}$ \\
\hline
\end{tabular}

en histologías agresivas (células altas, esclerosante difusa, etc.) mejora la supervivencia global; sin embargo, en estudios con pacientes con ganglios positivos, extensión extratiroidea microscópica o tumor mayor a $4 \mathrm{~cm}$, la supervivencia fue similar si los pacientes eran menores de 45 años de edad, aunque el tiempo de seguimiento no superó los siete años. Por otro lado, hubo ventaja en los mayores de 45 años que recibieron el tratamiento; aunque no superó el $73 \%$, fue superior al $69 \%$ del grupo que no lo recibió ${ }^{40}$.

Lamartina, et al. ${ }^{41}$ abonaron la confusión, ya que en la revisión de 13 estudios no aleatorizados no encontraron beneficio, mientras que 11 apoyaban su uso. Se requiere realizar más estudios, con criterios estandarizados y homogéneos.

Las dosis recomendadas para este grupo de pacientes son de 50 a $100 \mathrm{mCi}$; reservando dosis mayores para pacientes con más factores de riesgo, es decir, riesgo alto (véase mas adelante), aunque no hay estudios prospectivos que involucren este grupo de pacientes, una dosis de hasta $100 \mathrm{mCi}$ puede proveer el efecto terapéutico deseado, con la salvedad de administrar dosis mayores en caso de recurrencia.

\section{PACIENTES DE RIESGO ALTO}

Se recomienda la ablación rutinaria en pacientes de alto riesgo y se sugiere que la primera dosis de yodo 131 no supere los $150 \mathrm{mCi}$, en especial en los pacientes mayores de 45 años $^{42}$. Para el tratamiento de la recaída o persistencia se han propuesto diferentes enfoques. Se usaba el concepto de dosis fijas altas, es decir, de $100-150 \mathrm{mCi}$, si la persistencia o recaída estaba confinada al lecho tiroideo; de 150-175 mCi si había afección de los ganglios linfáticos; de 200-250 mCi si existían metástasis en los pulmones, y en caso de metástasis óseas se recurría a 200-300 $\mathrm{mCi}^{43}$. Sin embargo, la eficacia del yodo 131 está relacionada con la dosis de radiación liberada al tejido neoplásico y la radiosensibilidad del mismo, de manera que la radiosensibilidad resulta mayor en los jóvenes (< 45 años), con pequeñas metástasis (< $10 \mathrm{~mm})$, tumores bien diferenciados (papilar y folicular) y ávidos por el yodo 131 .

Hoy se reconoce que la dosis de radiación absorbida máxima tolerada (MTRD) es de 200 cGy en sangre ${ }^{44}$ y a mayor edad la función renal disminuye, como sucede en pacientes con insuficiencia renal o falla cardíaca, y con ello el aclaramiento del yodo 131 disminuye ${ }^{45,46}$. En este sentido, Tuttle, et al. $47^{75}$ demostraron que dosis empíricas de $140 \mathrm{mCi}$ excedían la MTRD en menos del $7 \%$ en pacientes menores de 70 años; este porcentaje se incrementaba al 13\% en mayores de 80 años; por otra parte, las dosis de 200 y $250 \mathrm{mCi}$ excedían la MTRD en menores de 70 años en el 15 y el $22 \%$, respectivamente, y en mayores de 70 años, hasta en el 38 y el $50 \%$, respectivamente. Sin embargo, es posible administrar dosis mayores a $200 \mathrm{mCi}$, siempre y cuando se realice un estudio dosimétrico previo y los pacientes sean menores de 65 años de edad.

Actualmente no se recomienda administrar yodo $131 \mathrm{en}$ una dosis superior a $200 \mathrm{mCi}$, ya que no se ha demostrado su superioridad; por el contrario, la exposición a radiación es mayor, los efectos secundarios son más frecuentes y prolongados, y la dosis acumulada es mayor sin que haya una mejor supervivencia.

\section{Utilidad del rastreo pre y postratamiento con yodo 131}

Algunos centros de medicina nuclear realizan un rastreo de cuerpo completo antes de recibir la dosis ablativa o terapéutica, para estimar la dosis de yodo 131 necesaria. Con la disponibilidad de equipos de SPECT/CT, se ha incrementado la sensibilidad y especificidad respecto al rastreo planar. La sensibilidad del rastreo planar alcanza el $41 \%$ y la especificidad, el $68 \%$, mientras que la sensibilidad del SPECT alcanza el $45 \%$ y la especificidad, el $89 \%$, pero con el SPECT/CT la sensibilidad se incrementa hasta el $50 \%$ y la especificidad alcanza el $100 \%{ }^{48}$.

Avram, et al., en una serie de 320 pacientes a los que se les practicó SPECT/CT preablación con yodo 131, reestadificaron al $4 \%$ de los pacientes menores de 45 años y at $25 \%$ de los mayores de 45 años $^{49}$. Con el uso generalizado de factores clínico-patológicos actualmente no se recomienda el rastreo preablación como estrategia para definir la dosis, pero puede ser necesario en pacientes en los que la enfermedad residual no haya sido evaluada con un estudio histopatológico o ultrasonido. 
El «aturdimiento» se define como una disminución en la captación de yodo 131 por las células tiroideas neoplásicas luego de la administración de una dosis diagnostica de yodo $131^{50}$. Puede deberse a la reducción en el número de células tiroideas funcionales debido a la muerte celular producida por la radiación $B$ del yodo 131 o a la disminución de la capacidad de las células tiroideas viables para atrapar o retener el yodo 131 durante un intervalo de tiempo. Guiraud-Vitaux, et al. ${ }^{51}$ apuntaron que puede existir necrosis infraterapéutica debido a altas dosis de yodo 131, lo cual implicaría una reducción de la eficacia de las dosis terapéuticas tras el diagnóstico por imagen. Sin embargo, la investigación de este fenómeno ha conducido a conclusiones contradictorias $^{52}$. Investigaciones recientes han demostrado que es un fenómeno radiobiológico estrechamente relacionado con la radiación absorbida, más que con la dosis administrada, y que el aturdimiento a menudo se observa $48 \mathrm{~h}$ después de una dosis de yodo $131^{53}$.

En otro orden de ideas, se recomienda practicar un rastreo corporal 5-8 días después de la ablación o dosis terapéutica, ya que identifica focos metastásicos adicionales en el $10-26 \%$ de los pacientes cuando se compara con un rastreo diagnóstico, aunque algunas series de estudios han reportado nuevos focos hasta en 31\%. El SPECT/CT mejora la sensibilidad hasta el $78 \%$ y la especificidad hasta el $100 \%$, reduciendo la necesidad de estudios seccionales en el $20 \%$ de los casos (Fig. 3) $)^{46}$.

\section{SEGUIMIENTO}

El rastreo de cuerpo completo más SPECT/CT con yodo 131 se usa en el seguimiento al menos seis meses después de la dosis para ablación o terapéutica (según la ATA) o al menos nueve meses (según la BTA) bajo tres escenarios: pacientes con captación anormal fuera del lecho tiroideo después del rastreo postratamiento o postablación; pacientes con escasa información en el rastreo postablación debido a grandes remanentes que impiden definir si ha existido captación fuera del lecho tiroideo y pacientes con presencia o aparición de novo de Ac-anti-Tg, aun en au sencia de hallazgos sospechosos de enfermedad residual por ultrasonido.

Los hallazgos clínicos, bioquímicos y de imagen deben conjuntarse durante el seguimiento para redefinir el tratamiento, otorgar la terapia más apropiada y definir la respuesta. Tuttle, et al. ${ }^{54}$ y Vaisman, et al. ${ }^{55}$ han contribuido a una mejor evaluación, definiendo cuatro categorías de respuesta:

- Respuesta excelente: se obtiene cuando no existe evidencia de enfermedad estructural (por imagen con ultrasonido o SPECT/CT) y clínica o bioquímica (Tg suprimida $<0.2 \mathrm{ng} / \mathrm{ml}$ o estimulada $<1 \mathrm{ng} / \mathrm{ml}$ ). Ocurre en el $86-91 \%$ de los pacientes con bajo riesgo, en el 57-63\% de los pacientes con riesgo intermedio y en el $14-16 \%$ de los pacientes con riesgo alto.

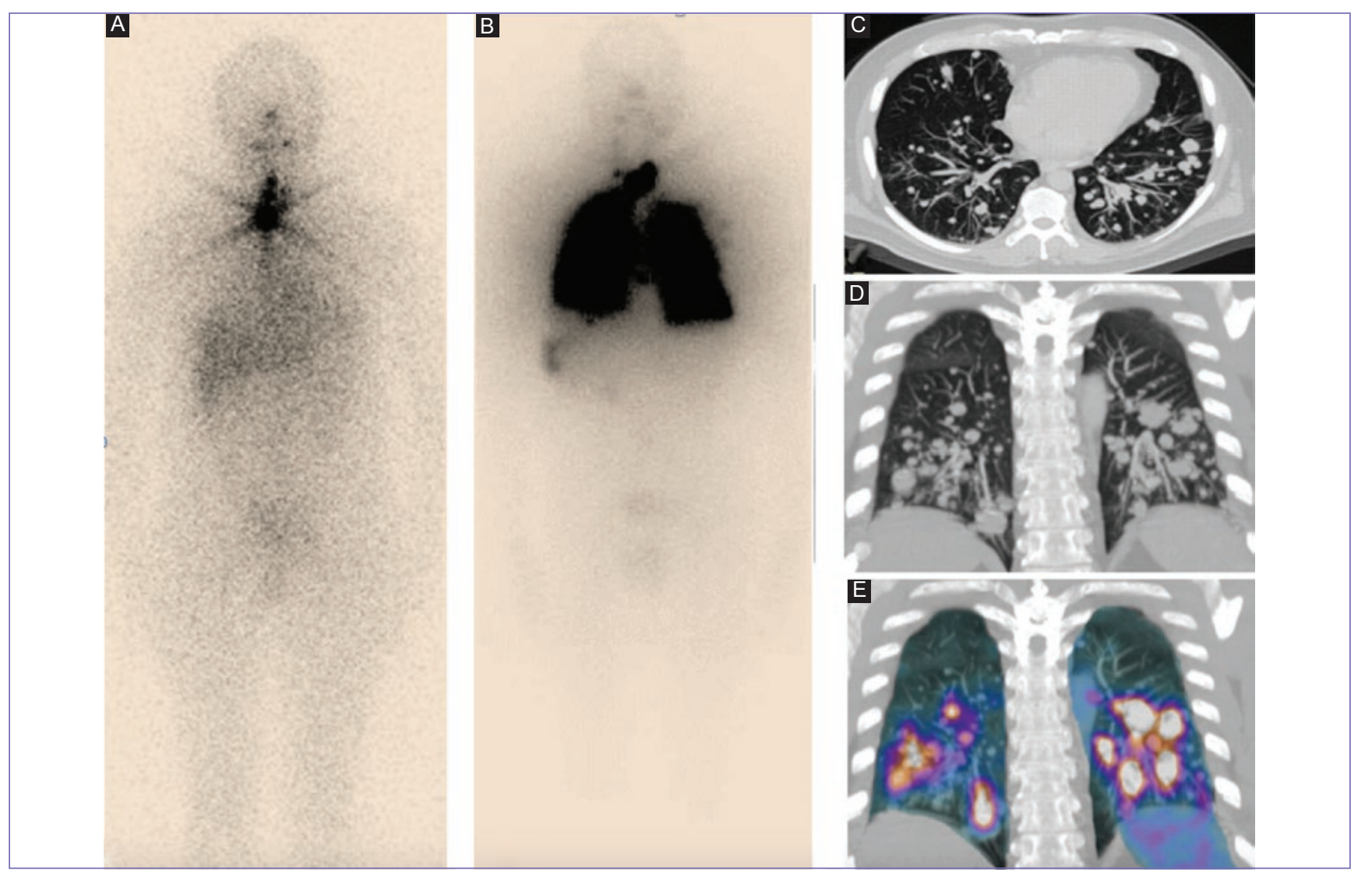

Figura 3. A: rastreo planar en proyección anterior practicado $48 \mathrm{~h}$ después de administrar 5 mCi de yodo 131 . Se observa una intensa captación focal anormal limitada al lecho tiroideo. Debido a la sintomatología respiratoria, se practica una TC sin contraste donde se observan múltiples imágenes nodulares en ambos pulmones compatibles con metástasis pulmonares (C y D); por ello, se decide administrar 200 mCi de yodo 131 y siete días después en el rastreo planar en proyección anterior se observa una intensa captación en el lecho tiroideo y en ambos pulmones (B). De forma posterior al rastreo, el SPECT/CT evidencia infiltrado pulmonar y captación de yodo 131, no visualizado en el rastreo planar preablación (E). 
- Respuesta bioquímica incompleta: resulta cuando existe un método de imagen negativo, pero niveles de $\mathrm{Tg}$ suprimida $>1 \mathrm{ng} / \mathrm{ml}$, Tg estimulada $>10 \mathrm{ng} / \mathrm{ml}$ o elevación de los Ac-anti-Tg. Esta respuesta la presentan el 11-19\% de los pacientes de bajo riesgo, el $21-22 \%$ de aquéllos con riesgo intermedio y el $16-18 \%$ de los de riesgo alto.

- Respuesta estructural incompleta: se refiere a aquellos pacientes con enfermedad estructural o funcional por cualquier método de imagen con cualquier nivel de $\mathrm{Tg}$ y/o Ac-anti-Tg; está presente en el 2-6\% de los pacientes con bajo riesgo, en el $19-28 \%$ de los pacientes con riesgo intermedio y en el $67-75 \%$ de aquéllos con riesgo alto.

- Respuesta indeterminada: se refiere a hallazgos inespecíficos en los estudios de imagen, niveles de $\mathrm{Tg}$ suprimida $<1 \mathrm{ng} / \mathrm{ml}$, niveles de $\mathrm{Tg}$ estimulada $<$ $10 \mathrm{ng} / \mathrm{ml}$ o niveles estables de Ac-anti-Tg. Esta respuesta está presente en el $12-29 \%$ de los pacientes de bajo riesgo, en el $8-23 \%$ de los pacientes con riesgo intermedio y en el $0-4 \%$ de los pacientes de riesgo alto.

No es suficiente realizar rastreos de cuerpo completo con SPECT/CT, sino que debe correlacionarse con los marcadores específicos, Tg y Ac-anti-Tg. Es bien conocido que las mediciones de los niveles de Tg son de particular importancia para monitorizar a los pacientes con enfermedad residual y recaída. En ausencia de Ac-anti-Tg, la Tg tiene una elevada sensibilidad y especificidad para documentar la recaída o persistencia de la enfermedad. Muchos autores recomiendan su medición con la TSH estimulada debido a que puede incrementar los niveles de Tg hasta 5-10 veces. Los niveles de $\mathrm{Tg}$ estimulados $<1 \mathrm{ng} / \mathrm{ml}$, en ausencia de Ac-anti-Tg, tienen una probabilidad del $98 \%$ para identificar pacientes con ausencia de la enfermedad ${ }^{56}$.

Por otro lado, los Ac-anti-Tg pueden estar presentes o aparecer de novo hasta en el $25 \%$ de los pacientes, en particular en aquéllos con tiroiditis de Hashimoto ${ }^{57}$. Las enfermedades autoinmunes tiroideas se asocian a la producción de anticuerpos en los linfocitos intratiroideos y después de una tiroidectomía total los niveles permanecen por años elevados sin evidencia de enfermedad. La razón es que en los ganglios linfáticos cervicales se inicia y disemina la respuesta autoinmune en respuesta a la de $\mathrm{Tg}$ en las células presentadoras de antígeno. La vida media de los Ac-anti-Tg después de una tiroidectomía total es de 10 semanas. Este decremento rápido se debe a la formación de complejos TgAc-anti-Tg en respuesta a la elevación de la Tg después de la cirugía ${ }^{58}$.

La cirugía puede iniciar o propiciar la aparición de novo de Ac-anti-Tg, que tienden a declinar en el curso de meses. Los pacientes pueden no alcanzar un estado negativo de Ac-anti-Tg durante el primer año postoperatorio e incluso exhibir un aumento (o la aparición de novo) durante los seis meses posteriores al tratamiento con yodo 131 cuando hay liberación de Tg secundaria a daño radiolítico del tejido tiroideo ${ }^{59}$.

Para un seguimiento eficaz de los Ac-anti-Tg a largo plazo, es fundamental utilizar un método sensible cada 6-12 meses. Kim, et al. ${ }^{60}$ encontraron que en pacientes con un descenso mayor al $50 \%$ de los niveles de Ac-anti-Tg en los 6-12 meses posteriores al yodo $131<1 \%$ desarrollaron una recaída; en cambio, el $19 \%$ de los pacientes con un descenso menor de $50 \%$ en el mismo intervalo presentaron una recaída, mientras que el $37 \%$ de los que incrementaron los niveles presentaron recaída.

\section{Rol del PET/CT con 2-[ $\left.{ }^{18} \mathrm{~F}\right]$-fluoro-2-deoxy-D- glucosa}

El PET/CT con 2-[ $\left.{ }^{18} \mathrm{~F}\right]$-fluoro-2-deoxy-D-glucosa (PET/CT= FDG) es una valiosa herramienta en pacientes con elevación de los niveles de $\mathrm{Tg}$ y/o Ac-anti-Tg y rastreo con yodo 131 negativo ${ }^{61}$. En las neoplasias tiroideas la captación del radio fármaco $18 \mathrm{~F}-\mathrm{FDG}$ se limita a los tumores más agresivos o de alto grado, con nula o escasa captación por los tumores bien diferenciados; a este fenómeno Feine, et al. ${ }^{62}$ lo denominaron fenómeno de flip-flop.

Debido a su carácter hidrófilo, la glucosa requiere proteínas transmembrana trasportadoras de glucosa (GLUT) que le permitan cruzar la membrana celular. La sobreexpresión de GLUT1 en la membrana celular de las neoplasias tiroideas está estrechamente relacionada con los tumores con un comportamiento biológico más agresivo. Además, la TSH juega un rol importante, ya que estimula el transporte de glucosa y la actividad glucolítica en los tirocitos a través de la translocación de GLUT1, así como la neosíntesis de GLUT1 por la activación de su expresión génica, lo cual indica que la sensibilidad del PET/CT-FDG está influenciada por los niveles de $\mathrm{TSH}^{63}$.

Salvatori, et al. ${ }^{64}$ sugieren que las indicaciones para practicar PET/CT-FDG se dividan en indicaciones fuertemente definidas y no completamente definidas. Las primeras in cluyen pacientes con niveles elevados de $\operatorname{Tg}(>10 \mathrm{ng} / \mathrm{ml})$ y estudio de imagen negativo (ultrasonido, rastreo con yodo 131). La elevación de Ac-anti-Tg o su aparición de novo pueden alterar drásticamente los valores de Tg. Por lo tanto, también es indicación la elevación de Ac-anti-Tg o la apari-ción de novo ante niveles de $\mathrm{Tg}<2 \mathrm{ng} / \mathrm{ml}$.

La importancia pronóstica del PET/CT-FDG radica en que la mayor parte de los pacientes con enfermedad metastásica tienen avidez por el 18F-FDG, lo que sugiere mayor agresividad, desdiferenciación y células metabólicamente activas, mientras que un PET/CT-FDG negativo predice un pronóstico favorable ${ }^{65}$. En un análisis multivariado practicado por Wang, et al. ${ }^{66}$ se analizaron 400 pacientes con características de alto riesgo y se observó que la edad y el PET/CT-FDG eran los factores pronóstico más importantes, ya que hubo una relación inversa entre la supervivencia y el número de lesiones metabólicamente activas.

La sensibilidad y la especificidad guardan relación con los niveles de Tg. En un metaanálisis realizado por Leboulleux, et al. ${ }^{67}$ se demostró que la sensibilidad y la especificidad están fuertemente influenciadas con un punto de corte de $\mathrm{Tg}>10 \mathrm{ng} / \mathrm{ml}$, y son del 83 y el $84 \%$, respectivamente, en pacientes con enfermedad no yodocaptante. Otro análisis demostró que la sensibilidad a niveles de $\mathrm{Tg}<10 \mathrm{ng} / \mathrm{ml}$, $>10$ pero $<100 \mathrm{ng} / \mathrm{ml} \mathrm{y}>100 \mathrm{ng} / \mathrm{ml}$ fue del 10.5 , el $75.6 \mathrm{y}$ el $91 \%$, respectivamente. Además, la sensibilidad también depende del lugar y tamaño de las metástasis. Para las metástasis ganglionares $<10 \mathrm{~mm}$ la sensibilidad es del $69.7 \%$ y la especificidad, del $83.3 \%$, mientras que para las mayores de $11 \mathrm{~mm}$ la sensibilidad es del $95.7 \%$ y la especificidad, del $80 \%$. Por otro lado, con metástasis pulmonares $<10 \mathrm{~mm}$ la 
sensibilidad es del $41 \%$ y la especificidad, del $80 \%$, mientras que para las mayores de $11 \mathrm{~mm}$ la sensibilidad es del $94 \%$ y la especificidad, del $89 \%{ }^{68}$.

Como mencionamos, la sensibilidad del SPECT/CT es mayor, por lo que, ante la ausencia de hallazgos con niveles elevados de Tg o Ac-anti-Tg, también se indica la necesidad de PET/CT-FDG (Fig. 4).
Debido a la reciente implementación en algunos centros de las mutaciones oncogénicas relacionadas con el BRAF (V600E), existen algunos estudios que demuestran la existencia de una correlación con el hipermetabolismo en los pacientes con dicha mutación ${ }^{69}$. En el estudio realizado por Nagarajah, et al. ${ }^{70}$, con una muestra de 48 pacientes con CDT, 24 de ellos con mutación del BRAF (V600E) y 24 BRAF-

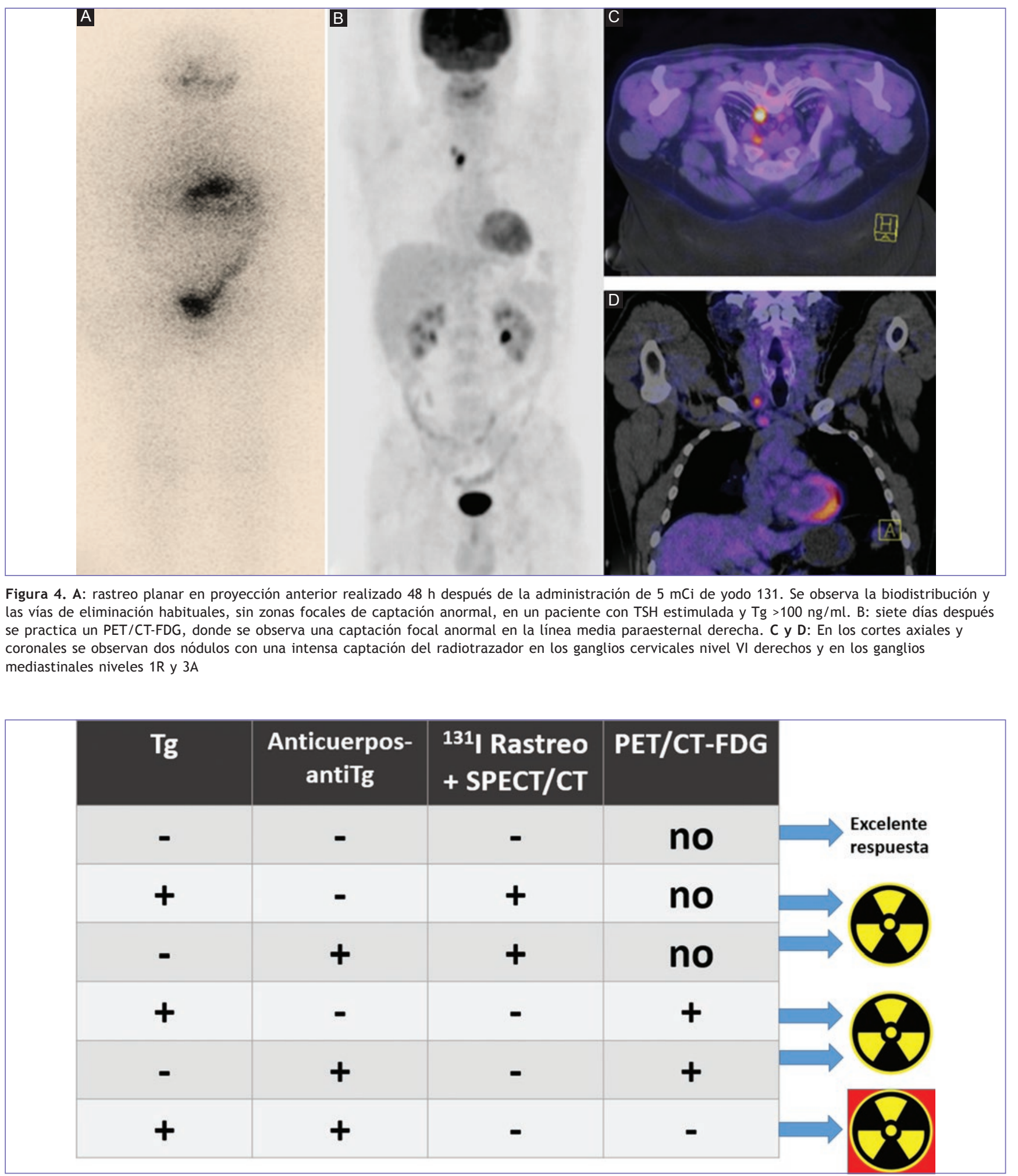

Figura 5. Recomendaciones según el escenario clínico posterior a la administración de radioyodo. 
wild type, se observó una correlación significativamente mayor de la actividad metabólica en los pacientes con la mutación del BRAF (V600E) y se concluyó que una tasa metabólica alta puede estar relacionada con la mutación del gen, lo que conlleva mayor agresividad tumoral y peor pronóstico; sin embargo, se necesitan más estudios para correlacionar estos hallazgos.

Entre las indicaciones no completamente definidas se incluye la práctica de PET/CT en pacientes con enfermedad refractaria, para evaluar la respuesta al tratamiento con terapias blanco, en la estadificación y seguimiento de histologías de alto riesgo, en nódulos tiroideos indeterminados después de una biopsia por aspiración y recientemente para cirugía radioguiada ${ }^{71}$.

Para ilustrar mejor el seguimiento y tratamiento de los pacientes con CDT manejados con yodo 131, véase la (Fig. 5).

\section{AGRADECIMIENTOS}

A la Dra. Claudia Mosqueda y a la Dra. Angélica Arellano, por su colaboración en la generación y edición de las ilustraciones.

\section{BIBLIOGRAFÍA}

1. Ferlay J, Soerjomataram I, Ervik M, et al. GLOBOCAN 2012 v1.0, Cancer Incidence and Mortality Worldwide: IARC Cancer Base No. 11. Lyon, France: International Agency for Research on Cancer; 2013. [Internet] Disponible en: http://globocan.iarc.fr, accessed on 20/December/2016.

2. Bongiovanni M, Paone G, Ceriani L, Pusztaszeri M. Cellular and molecular basis for thyroid cancer imaging in nuclear medicine. Clin Transl Imaging. 2013;1:149-61.

3. Granados García M, León Takahashi AM, Guerrero Huerta FJ, Taissoun Aslan ZA. [Differentiated thyroid cancer: an ancient disease with new knowledge]. Gac Med Mex. 2014;150(1):65-77.

4. Robbins RJ, Schlumberger MJ. The evolving role of $131 \mathrm{i}$ for the treatment of differentiated thyroid carcinoma. J Nucl Med. 2005;46 Suppl $1: 28 \mathrm{~S}-37 \mathrm{~S}$.

5. Maxon HR, Thomas SR, Hertzberg VS, et al. Relation between effective radiation dose and outcome of radioiodine therapy for thyroid cancer. N Engl J Med. 1983;309(16):937-41.

6. Braunstein GD, ed. Thyroid Cancer. Volume 32 of Endocrine Updates. EE.UU.: Springer Science \& Business Media; 2011.

7. Dorn R, Kopp J, Vogt H, Heidenreich P, Carroll RG, Gulec SA. Dosimetry-guided radioactive iodine treatment in patients with metastatic differentiated thyroid cancer. Largest safe dose using a risk-adapted approach. J Nucl Med. 2003;44(3):451-56.

8. Eskandari S, Loo DD, Dai G, Levy O, Wright EM, Carrasco N. Thyroid $\mathrm{Na}+/$ I-symporter. J Biol Chem. 1997;272(43):27230-8.

9. Vieja ADL, Dohan O, Levy O, Carrasco N. Molecular analysis of the sodium/iodide symporter: impact on thyroid and extrathyroid pathophysiology. Physiol Rev. 2000;80(3):1083-105.

10. Caillou B, Troalen F, Baudin E, et al. $\mathrm{Na}+/ \mathrm{I}-$ symporter distribution in human thyroid tissues: an immunohistochemical study. J Clin Endocrinol Metab. 1998;83(11):4102-6.

11. Daniels GH, Haber DA. Will radioiodine be useful in treatment of breast cancer? Nat Med. 2000;6(8):859-60.

12. Filetti S, Bidart JM, Arturi F, Caillou B, Russo D, Schlumberger M. Sodium/iodide symporter: a key transport system in thyroid cancer cell metabolism. Eur J Endocrinol. 1999;141(5):443-57.

13. Dohan O, Baloch Z, Banrevi Z, Livolsi V, Carrasco N. Predominant intracelular overexpression of the $\mathrm{Na}+/ \mathrm{I}+$ symporter (NIS) in a large sampling of thyroid cancer cases. J Clin Endocrinol Metab. 2001; 86(6):2697-700.
14. Saito T, Endo $\mathrm{T}$, Kawaguchi $\mathrm{A}$, et al. Increased expression of the sodium/iodide symporter in papillary thyroid carcinomas. J Clin Invest. 1998;101(7):1296-300.

15. Min JJ, Chung JK, Lee YJ, et al. Relationship between differentiation and expression of sodium/iodide symporter or glucose transporter-1 in differentiated thyroid carcinoma [abstract]. J Nucl Med. 2001;42(su-

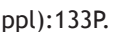

16. Wapnir IL, van de Rijn M, Nowels K, et al. Immunohistochemical profile of the sodium/iodide symporter in thyroid, breast, and other carcinomas using high-density tissue microarrays and conventional sections. J Clin Endocrinol Metab. 2003;88(4):1880-8.

17. Sawka AM, Ibrahim-Zada I, Galacgac P, et al. Dietary iodine restriction in preparation for radioactive iodine treatment or scanning in well-differentiated thyroid cancer: a systematic review. Thyroid. 2010 20(10):1129-38.

18. Thyroid cancer. In National Comprehensive Cancer Network (NCCN) Clinical Practice Guidelines in Oncology, version 2.2016. National Com prehensive Cancer Network.

19. Haugen BR, Alexander EK, Bible KC, et al. American Thyroid Association Management Guidelines for Adult Patients with Thyroid Nodules and Differentiated Thyroid Cancer. Thyroid. 2016;26(1):1-133.

20. Golger A, Fridman T, Eski S, Witterick IJ, Freeman JL, Walfish PG: Three-week thyroxine withdrawal thyroglobulin stimulation screening test to detect low-risk residual/recurrent well-differentiated thyroid carcinoma. J Endocrinol Invest. 2003;26(10):1023-31.

21. Dow KH, Ferrell B, Anello C. Quality-of-life changes in patients with thyroid cancer after withdrawal of thyroid hormone therapy. Thyroid. 1997;7(4):613-9.

22. Pérez $B$, Almeda $P$, Arechavaleta $M$, et al. Guía clínica para el diagnóstico y tratamiento del cáncer diferenciado de tiroides. Sociedad Mexicana de Nutrición y Endocrinología. 2009.

23. Potzi C, Moameni A, Karanikas G, et al. Comparison of iodine uptake in tumour and nontumour tissue under thyroid hormone deprivation and with recombinant human thyrotropin in thyroid cancer patients. Clin Endocrinol. 2006;65(4):519-23.

24. Rosário PW, Borges MA, Purisch S. Preparation with recombinant human thyroid-stimulating hormone for thyroid remnant ablation with $131 \mathrm{I}$ is associated with lowered radiotoxicity. J Nucl Med. 2008;49(11):1776-82.

25. Leboeuf R, Perron P, Carpentier AC, Verreault J, Langlois MF. L- $† 3$ preparation for whole-body scintigraphy: a randomized controlled trial. Clin Endocrinol (Oxf). 2007;67(6):839-44.

26. Pluijmen MJ, Eustatia-Rutten C, Goslings BM, et al. Effects of low-iodide diet on postsurgical radioiodide ablation therapy in patients with differentiated thyroid carcinoma. Clin Endocrinol (Oxf). 2003;58:428-35.

27. Sonenberg M. Low-lodine Diet in the Treatment of Differentiated Thyroid Cancer with Radioactive lodine. Endocrine. 2002;17(2):141-3

28. Robbins RJ1, Driedger A, Magner J; U.S. and Canadian Thyrogen Compassionate Use Program Investigator Group. Recombinant human thyrotropin-assisted radioiodine therapy for patients with metastatic thyroid cancer who could not elevate endogenous thyrotropin or be withdrawn from thyroxine. Thyroid. 2006;16(11):1121-30.

29. Liel Y. Preparation for radioactive iodine administration in differentiated thyroid cancer patients. Clin Endocrinol (Oxf). 2002;57(4):523-7.

30. Van Nostrand D, Wartofsky L. Radioiodine in the treatment of thyroid cancer. Endocrinol Metab Clin North Am. 2007;36(3):807-22.

31. Perris P, Colley S, Boelaert K, et al. British Thyroid Association Guidelines for the Management of Thyroid Cancer. Clin Endocrinol: 2014;81(Supp 1):1-122.

32. Carty SE, Doherty GM, Inabnet WB III, et al. American Thyroid Association statement on the essential elements of interdisciplinary com munication of perioperative information for patients undergoing thyroid cancer surgery. Thyroid. 2012;22(4):395-9.

33. Verkooijen RB, Verburg FA, van Isselt JW, Lips CJ, Smit JW, Stokkel MP The success rate of $\mathrm{I}-131$ ablation in differentiated thyroid cancer: comparison of uptake-related and fixed-dose strategies. Eur J Endocrinol. 2008;159(3):301-7.

34. Robbins $R$, Schlumberger $M$. The evolving role of $131 \mathrm{i}$ for the treatment of differentiated thyroid carcinoma. J Nucl Med. 2005;46 Suppl $1: 28 \mathrm{~S}-37 \mathrm{~S}$.

35. Schvartz C, Bonnetain F, Debakuyo S, et al. Impact on overall survival of radioactive iodine low-risk differentiated thyroid cancer patients. J Clin Endocrinol Metab. 2012;97(5):1526-35.

36. Mallick U, Harmer C, Yap B, et al. Ablation with low-dose radioiodine and thyrotropin alfa in thyroid cancer. N Engl J Med. 2012; 366(18):1674-85 
37. Cheng W, Ma C, Fu H, et al. Low- or High-Dose Radioiodine Remnant Ablation for Differentiated Thyroid Carcinoma: A Meta-Analysis. J Clin Endocrinol Metab, 2013;98(4):1353-60.

38. Fallahi B, Beiki D, Takavar A, et al. Low versus high radioiodine dose in postoperative ablation of residual thyroid tissue in patients with differentiated thyroid carcinoma: a large randomized clinical trial. Nucl Med Commun. 2012;33(3):275-82.

39. Shah MD, Hall FT, Eski SJ, Witterick IJ, Walfish PG, Freeman JL. Clinical course of thyroid carcinoma after neck dissection. Laryngoscope. 2003;113(12):2102-7.

40. Ruel E, Thomas S, Dinan M, Perkins JM, Roman SA, Sosa JA. Adjuvant radioactive iodine therapy is associated with improved survival for patients with intermediate-risk papillary thyroid cancer. J Clin Endocrinol Metab. 2015;100(4):1529-36.

41. Lamartina L, Durante C, Filetti S, Cooper DS. Lowrisk differentiated thyroid cancer and radioiodine remnant ablation: a systematic review of the literature. J Clin Endocrinol Metab. 2015;100(5):1748-61.

42. Sabra M, Grewal R, Ghossein RM, Tuttle RM. Higher administered activities of radioactive iodine are associated with less structural persistent response in older, but not younger, papillary thyroid cancer patients with lateral neck lymph node metastases. Thyroid. 2014; 24(7):1088-95.

43. Schlumberger $M$, Tubiana $M$, De Vathaire $F$, et al. Long-term results of treatment of 283 patients with lung and bone metastases from differentiated thyroid carcinoma. J Clin Endocrinol Metab. 1986;63(4):960-7.

44. Van Nostrand D, Atkins F, Yeganeh F, Acio E, Bursaw R, Wartofsky L. Dosimetrically determined doses of radioiodine for the treatment of metastatic thyroid carcinoma. Thyroid. 2002;12(2):121-34.

45. Beckers C, van Ypersele de Strihou C, Coche E, Troch R, Malvaux P. lodine metabolism in severe renal insufficiency. J Clin Endocrinol Metab. 1969;29(2):93-6.

46. Ciappuccini R, Heutte N, Trzepla G, et al. Postablation (131)I scintigraphy with neck and thorax SPECT-CT and stimulated serum thyroglobulin level predict the outcome of patients with differentiated thyroid cancer. Eur J Endocrinol. 2011;164(6):961-9.

47. Tuttle $M$, Lebeauf $R$, Robbins $R$, et al. Empiric Radioactive lodine Dosing Regimens Frequently Exceed Maximum Tolerated Activity Levels in Elderly Patients with Thyroid Cancer. J Nucl Med. 2006;47(10): 1587-91.

48. Avram AC. Radioiodine Scintigraphy with SPECT/CT: An Important Diagnostic Tool for Thyroid Cancer Staging and Risk Stratification. J Nucl Med. 2012;53(5):754-64.

49. Avram AM, Doherty GM, Fig LM, Wong K. Diagnostic 131-I fusion SPECTCT Imaging in postoperative thyroid cancer patients: what is the impact on staging? [abstract]. Thyroid. 2011;21(Suppl 1):193.

50. Brenner W. Is Thyroid Stunning a Real Phenomenon or Just Fiction? J Nucl Med. 2002;43(6):835-6.

51. Guiraud-Vitaux F, Feldmann G, Vadrot N, et al. Early ultrastructural injuries in the thyroid of the normal rat radioinduced by diagnostic and/or therapeutic amounts of iodine-131. Cell Mol Biol (Noisy-legrand). 2001;47(3):495-502.

52. Kao $\mathrm{CH}$, Yen TC. Stunning effects after a diagnostic dose of iodine-131. Nuklearmedizin. 1998;37(1):30-2.

53. Aide N, Heutte N, Rame JP, et al. Clinical relevance of single-photon emission computed tomography/computed tomography of the neck and thorax in postablation 131/ scintigraphy for thyroid cancer. J Clin Endocrinol Metab. 2009;94(6):2075-84.

54. Tuttle RM, Tala $\mathrm{H}$, Shah J, et al. Estimating risk of recurrence in differentiated thyroid cancer after total thyroidectomy and radioactive iodine remnant ablation: using response to therapy variables to modify the initial risk estimates predicted by the new American Thyroid Association staging system. Thyroid. 2010;20(12):1341-9.

55. Vaisman F, Shaha A, Fish S, Michael Tuttle R. Initial therapy with either thyroid lobectomy or total thyroidectomy without radioactive iodine remnant ablation is associated with very low rates of structural disease recurrence in properly selected patients with differentiated thyroid cancer. Clin Endocrinol (Oxf). 2011;75(1):112-9.

56. Webb RC, Howard RS, Stojadinovic A, et al. The utility of serum thyroglobulin measurement at the time of remnant ablation for predicting disease-free status in patients with differentiated thyroid cancer: a metaanalysis involving 3947 patients. J Clin Endocrinol Metab? 2012;97(8):2754-63.

57. Latrofa F, Ricci D, Montanelli L, et al. Lymphocytic thyroiditis on histology correlates with serum thyroglobulin autoantibodies in patients with papillary thyroid carcinoma: impact on detection of serum thyroglobulin. J Clin Endocrinol Metab. 2012;97(7):2380-7.

58. Görges R, Maniecki M, Jentzen W, et al. Development and clinicaE impact of thyroglobulin antibodies in patients with differentiated thyroid carcinoma during the first 3 years after thyroidectomy. Eur $\mathrm{J}$ Endocrinol. 2005;153(1):49-55.

59. Spencer CA. Clinical review: Clinical utility of thyroglobulin antibody (TgAb) measurements for patients with differentiated thyroid cancers (DTC). J Clin Endocrinol Metab. 2011;96(12):3615-27.

60. Kim WG, Yoon JH, Kim WB, et al. Change of serum antithyroglobulin antibody levels is useful for prediction of clinical recurrence in thyroglobulin-negative patients with differentiated thyroid carcinoma. Clin Endocrinol Metab. 2008;93(12):4683-9.

61. Palaniswamy SS, Subramanyam P. Diagnostic utility of PETCT in thyroid malignancies: an update. Ann Nucl Med. 2013;27(8):681-93.

62. Feine U, Lietzenmayer R, Hanke JP, Held J, Wöhrle H, Müller-Schauenburg W. Fluorine-18-FDG and iodine-131-iodide uptake in thyroid cancer. J Nucl Med. 1996;37(9):1468-72.

63. Schonberger J, Ruschoff J, Grimm D, et al. Glucose transporter 1 gene expression is related to thyroid neoplasms with an unfavorable prog. nosis: an immunohistochemical study. Thyroid. 2002;12(9):747-54.

64. Salvatori M, Biondi B, Rufini V. 2-[18F]-fluoro-2-deoxy-D-glucose positron emission tomography/computed tomography in differentiated thyroid carcinoma: clinical indications and controversies in diagnosis and follow-up. Eur J Endo. 2016;173(3):115-30.

65. Pacak K, Eisenhofer G, Goldstein DS. Functional imaging of endocrine tumors: role of positron emission tomography. Endoc Rev. 2004; 25(4):568-80.

66. Wang W, Larson SM, Fazzari M, et al. Prognostic value of [18F]fluorodeoxyglucose positron emission tomographic scanning in patients with thyroid cancer. J Clin Endocrinol Metab. 2000;85(3):1107-13.

67. Leboulleux S, Schroeder PR, Schlumberger M, Ladenson PW. The role of PET in follow-up of patients treated for differentiated epitheliat thyroid cancers. Nat Clin Pract Endocrinol Metab. 2007;3(2):112-21.

68. Grabellus F, Worm K, Schmid KW, Sheu SY. The BRAFV600E mutation in papillary thyroid carcinoma is associated with glucose transporter 1 overexpression. Thyroid. 2012;22(4):377-82.

69. Xing M, Alzahrani AS, Carson KA, et al. Association between BRAF V600E mutation and mortality in patients with papillary thyroid cancer. JAMA. 2013;309(14):1493-501.

70. Nagarajah J, Ho AL, Tuttle RM, et al. Correlation of BRAFV600E Mutation and Glucose Metabolism in Thyroid Cancer Patients: An 18F-FDG PET Study. J Nucl Med. 2015;56(5):662-66.

71. Stokkel M, Duchateau C, Drogoiescu C. The value of FDG-PET in the follow-up of differentiated thyroid cancer: a review of the literature. Q J Nucl Med Mol Imag. 2006;50(1):78-87. 